\title{
Editoriali
}

\section{Assessing quality of life in people living with psychosis}

\author{
HELEN HERRMAN
}

The recent Global Burden of Disease studies (Murray \& Lopez, 1996) using a new index summating the death and the morbidity resulting from identifiable diseases, estimate that mental disorders contribute a high proportion of the burden of disease in all parts of the world. The focus will now move to demonstrating how much of the burden of disease can be relieved by service provision. Health care resources are limited and demand will continue to grow. The effectiveness of service provision for people living with psychosis as demonstrated by studies of patient outcomes will eventually be compared with the effectiveness of service provision for people with other illnesses (Andrews, 1999). However information about effectiveness can easily be misleading, and more work is needed before this comparison can be used meaningfully to allocate resources.

Assessing the burden averted by service provision, or the effectiveness of services, requires attention to the methods and types of outcome assessments, and to study design. Longitudinal studies of patient cohorts are required (Abrahamson, 1993), with assessment of outcomes on several dimensions (Thornicroft \& Tansella, 1996).

The measurement of treatment outcomes may be misleading unless it includes subjective quality of life (QOL) and disability as well as symptoms and death rates. The use of appropriate QOL measures is important in highlighting needs and outcomes in areas that are si-

Indirizzo per la corrispondenza: Professor H. Herrman, St. Vincent's Mental Health Service (Melbourne), 41 Victoria Parade, Fitzroy 3065, Victoria (Australia).

Fax: +61-3-9288.4802

E-mail: herrmahe@svhm.org.au gnificant to individuals and their families and which may not be addressed or demonstrated otherwise (Lehman, 1996). Few people other than those directly involved can describe how life is affected by a psychotic disorder. The effects of the illness and the consequent disability are often hidden, or may be wrongly attributed by others to laziness or life choices.

The need for adequate and continuing treatments in a stable, safe and stimulating environment for people with persisting psychotic disorders, and the efficacy of these, has been known to the field and demonstrated by cohort studies over some time. However there is much concern that the knowledge about efficacious treatments is not disseminated and applied (Davidson \& McGlashan, 1997). This requires the demonstration that treatments are effective in improving QOL, disability and symptoms in cohorts of patients in regular service environments. Otherwise, "there is pressure to repeat old mistakes in new settings, and to neglect long-term patients living in hospital and community settings in the face of opportunities for providing services to people with apparently more to gain" (Abrahamson, 1993).

\section{DEFINING QUALITY OF LIFE (QOL)}

QOL assessment is now commonly understood to have several dimensions and to require a subjective report, and may be carried out and recorded in a number of ways, for different purposes. Far from replacing other measures, it is one of a number of relevant measures when treating a patient or planning a health service. Two important issues determine the type of enquiry into QOL. The first is whether it should be done in a broad generic way, 
or else focus on the effects of a specific disease or circumstance. The second is whether the enquiry should be an objective or professional assessment of the patient and the patient's environment or whether it should be subjective, relying on the patient as the expert (Orley et al., 1998; Katschnig, 1996).

The World Health Organization (WHO) has defined QOL as individuals' perception of their position in life in the context of the culture and value systems in which they live and in relation to their goals, expectations, standards and concerns. It is a broad concept, incorporating the persons' physical health, psychological state, level of independence, social relationships, personal beliefs and relationship to salient features of the environment (Sartorius, 1990). It is firmly in the 'generic' and 'subjective' camp. It is the basis of recent work by WHO to develop a quality of life assessment instrument (the WHOQOL) applicable to people with different conditions and circumstances and across cultures (WHOQOL Group, 1998a, b). Two self-report versions of the instrument are available, one with 100 and one with 26 items, arranged in the above six domains. Both are available in several languages. A striking result of the project is that the national versions of the instrument are very similar. It seems possible that different national and cultural groups share a set of core concepts about QOL. Conditions and behaviours obviously differ across cultures, but subjective perception is not a linear reflection of these, and human perception is the most important common factor in understanding QOL (M. Bullinger, personal comunication).

Defining QOL as subjective, or as an internal experience, brings a degree of unity to the evaluation, within an individual as well as across cultures. QOL is influenced by current events and the environment, but also coloured by a person's past experiences, mental state, personality and expectations (Orley et al., 1998). It can be considered as a part of a person's mental state. However it will not emerge from a description of moods, behaviours and cognitions, but rather by asking subjects how bothered they are by any problems or how satisfied they are with aspects of their lives. The questions do not, for example, ask how long people sleep or whether they have continuing hallucinations, but how satisfied they are with their sleep or how bothered they are by unusual experiences. Just as several elements of the mental state are seen to rest on reactions to internal or external cues and circumstances which may vary from hour to hour, and many to rest equally heavily on underlying features of a person's personality or temperament, so there are influences of various types on the QOL. The WHOQOL project for instance has defined a set of these 'important' aspects. QOL can be thought of as a relatively stable internal state influenced by personal traits and by the surroundings and circumstances in which a person finds him or herself.

QOL is sometimes described as a unitary concept similar to satisfaction, happiness or well-being. However these states may usefully be considered as among, or even as resting on, several components of life experience all of which contribute to QOL. QOL may be seen as a multi-dimensional state which will usually remain stable over short time periods for a person, and will change gradually even in the face of dramatic changes in life circumstances or events.

Certain specific features such as the symptoms of a disease will have an effect on QOL, usually because they affect one of the core features. Whether particular questions are required for certain groups of people, possibly with certain illnesses, can be seen as a matter for investigation. While it is likely that specific questions about symptoms or medication side effects, for instance, will be more sensitive or responsive to change, that does not mean that they are better items for assessing QOL. If they are in some way included in the assessment, then they can be assessed by questions which explore how much they bother the subject, rather than whether they are present (Awad \& Hogan, 1994).

\section{FOUR CRITICAL QUESTIONS IN ASSESSING THE SUBJECTIVE QOL OF PEOPLE LIVING WITH PSYCHOSIS}

1. Clinicians may have reservations about accepting the validity of the responses about QOL from a person whose judgement is disturbed, because of delusions or thought disorder, or because of depression (Atkinson $e t$ al., 1997). Nevertheless, if QOL is accepted as subjective, then a patient's viewpoint is logically valid (Orley et al, 1998). Most people consider it likely that religious or political beliefs, for instance, will have an effect on a person's QOL. In either case the beliefs will be among a number of influences on QOL, and their effect on QOL cannot be discounted. Ultimately the question of the validity or meaning of patients' responses will be answered empirically with cross-sectional and longitudinal studies assessing the QOL of people living with psychosis.

Strauss (1989) has noted in another context that clinicians rely on patients' reports of their experiences of symptoms. These reports about delusions and hallucinations, for example, are the basis of diagnoses and the 
development of aetiological ideas. The subjective response of patients to anti-psychotic medication can be assessed consistently (Voruganti et al., 1998) and has prognostic value (Awad \& Hogan, 1994), and the assessment of depression or suicidality in a person with schizophrenia depends largely on listening to their own reports.

2. Conversely, is enquiry about the QOL redundant in a person affected by a severe mental illness? Subjective QOL does seem to be distinct from such clinical syndromes and mental states as depression and anxiety. In a study of patients with severe mental illness, it was found that measures of QOL are independently affected by clinical symptoms, especially depression and anxiety, and by age, marital status, social function and support, insight and intelligence level. Patients with larger and more satisfactory networks of social support report a better QOL (Corrigan \& Buican, 1995). Evidence about the relationship of QOL to psychotic symptoms is mixed. However attributing one's problems to a mental illness has been associated with reduced subjective QOL among persons diagnosed with schizophrenia (Mechanic et al., 1994). Similarly, more intelligent patients have reported a lower QOL (Skantze et al., 1992).

3. Another criticism of the subjective approach to assessing QOL among people with persisting mental illnesses is that they may perceive the quality of their lives differently from the way that social norms would predict. For instance, people living in institutions are sometimes seen as inappropriately content or resigned. It may be that changed expectations or "adaptation" lead them to assess their QOL as good. Expectations do indeed affect how a person views the quality of their life (Atkinson et al., 1997).

However the QOL of people living with psychosis, across studies using a variety of methods, is reported as worse than that of the general population and that of physically ill patients (Bobes \& Gonzalez, 1997). Furthermore, in a longitudinal study of long-term patients in London before and after deinstitutionalisation (Leff $e t$ al., 1994), most wanted to stay where they were five years after moving to community homes. While they were in hospital most wanted to leave. This questions the view that institutionalised patients would rate their QOL as good, and that patients were likely to be less happy outside institutions.

On the other hand, studies of subjective QOL especially when the results contrast with the expectations of professionals can give valuable information for clinical management and planning (Lehman, 1996). Recent work from Scandinavia is illustrative. When living standards were ensured by a well functioning social service system, the perceptions by the patients treated for schizophrenia outside hospital of their own QOL and their own standard of living were independent of each other. Despite good housing and an adequate income, 'inner experiences' were frequently reported as unsatisfactory, and differences in QOL were found across patients' age, education, and work status (Skantze et al., 1992). Ratings by clinicians and patients are more likely to coincide on clinical areas, such as symptoms and function, and less likely to agree on social and occupational aspects of QOL (Sainfort et al., 1996). These aspect of life are also potentially modifiable by interventions designed to improve subjective QOL (Trauer et al., 1998).

Failure to address an area of life with which a patient is dissatisfied can adversely affect the treatment alliance. Conversely, patients are unlikely to be motivated to change circumstances with which they are content even if the clinician and family feel otherwise (Lehman, 1996). QOL assessments are also relevant to family caregivers (Szmukler et al., 1996) and can provide similar guides to negotiating service goals with the patient and clinicians.

4. How well do QOL measures function as valid and sensitive indicators of mental health services?

Barry \& Crosby (1996) report that dramatic changes in clients' lives following resettlement are not captured by life satisfaction measures. Improvements in the QOL in their study of resettlement were evident in the objective quality of life indices, the qualitative data and other outcome measures, but were not reflected in the satisfaction ratings which remained relatively stable. While the lack of change in the satisfaction measures could reflect insensitivity of the measures, the findings of Skantze and colleagues noted above remind us that subjective changes may not follow even major changes to external circumstances, and so emphasise the importance of enquiring into each of these areas. The multidimensional nature of subjective QOL is also relevant here, with life satisfaction being one component. Additional and complementary measures will be required to assess changes in symptoms, functional activity and the environment, and in the subjective response across several dimensions of experience.

\section{SPECIFIC AND 'GENERIC' INSTRUMENTS}

The QOL measures used to date to help plan services and assess the effects of interventions (Barry \& Zissi, 1997) are mostly specific instruments for people diagnosed with schizophrenia (Lehman, 1996; Oliver et al., 1997). 
These include objective as well as subjective ratings of various dimensions of QOL.

In contrast, the use of a generic instrument such as the WHOQOL allows the effects of different disorders, whether mental or physical, to be compared. It can also remind the clinician of the effect of the mental disorder or side effects on the person's QOL. Specific questions related to the mental disorder will increase the sensitivity of the instrument to change in that condition in the short term, but will not allow a balanced assessment of the several aspects of overall QOL. A generic QOL instrument will often be used alongside measures of symptoms, side effects and disability.

\section{PROFILE AND INDEX SCORES}

An instrument producing a QOL profile, giving scores across several dimensions, is the preferred approach when managing individual patients and in broader service evaluations. However in some situations a single score or index is required to represent the value or utility of a given health outcome (Wilkinson et al., 1992). This is particularly important when mental health services need to justify resource use alongside other health services. Utility or preference measures which enable QALY calculation provide such an index. Their use in resource allocation and service and treatment evaluation is fervently debated (Chisholm $e t$ al., 1997). They are seen as important aids to planning and priority setting, but also criticised on technical and ethical grounds.

Existing utility or preference instruments give an insensitive measure of the outcomes of mental health care. They tend to give a much higher weighting to physical disability than to social disability and distress (Wilkinson et al., 1992). They may also fail to allow for the different valuations that people tend to give a health state depending upon the time spent in it. The chronicity of many mental disorders is likely to mean the undervaluing of associated disability (Kavanagh \& Stewart, 1995). However abandoning this approach in mental health runs the risk of marginalising people with mental illness in the resource allocation process (Chisholm et al., 1997). The development of indices more sensitive to the experience of people living with mental disorders is a priority.

This requires the use of suitable generic assessment instruments in empirical study of the profile of QOL scores in patients with various types, duration and severity of disorder, comparing people with mental di- sorders with those with physical disorders and those who are well. Evidence of this type, obtained in crosssection as well as over time, is eventually required to support the various judgements of relative health states that so far inform the construction of utility measures. The development of multi-attribute utility measures has used elements of this approach (Revicki, 1996; Hawthorne et al., 1999). Adding cost information to a valid index would allow different treatments to be compared in various ways in terms of their relative value for money. To be meaningful, these comparisons would need to take account of gradual changes in QOL or the maintenance of a given level of subjective $Q O L$ (Jenkins. 1996), which may be the desirable outcome for some people with persisting disorders.

The best forms of assessment will vary with the evaluation question. Measures providing a profile of scores for each patient give comprehensive information on the impact of treatments. These can be supplemented by summary indicators, such as utility scores or QALYs, which are necessary for economic analysis. These indicators, however, may hide outcomes important to patients or to clinicians. A similar situation is represented by the different approaches and uses of the single and multi-dimensional assessment of intelligence, or the rating of the likelihood of mental disorder with a single score on a General Health Questionnaire as compared with a clinical diagnostic assessment or a profile of symptoms.

\section{CONCLUSIONS}

QOL assessment assists in understanding the experience of people living with psychosis and their families. This perspective can then be added more clearly and rigorously to clinical management, including training and continuing education, and the planning of mental health services. To obtain a complete picture of a patient's condition, requires disability and QOL measures in addition to symptom measures. The concepts of impairment and disability as used by the World Health Organization (WHO) over the last two decades (Üstün \& Chatterji, 1998) have helped to define the elements of a broad clinical assessment in psychiatry. The recent development of the WHOQOL is complementary.

Clinicians are sometimes concerned about the validity and usefulness of QOL assessment in affected individuals. However these concerns are being addressed by empirical studies, and the place of QOL assessment in both epidemiological and clinical studies is

Epidemiologia e Psichiatria Sociale, 9, 1, 2000 
becoming established. Eventually we may look to a better understanding of how QOL assessments change over the course of psychotic and other mental disorders and in different treatment conditions (Russo et al., 1997); as well as how these data compare with those from other population groups, including those with physical disorders and the general population (Lehman, 1996).

Mental health professionals are being encouraged to take more notice of what patients think about their problems and treatment. The views of patients and families are now incorporated in good measures of disability and QOL to assist in understanding needs, gaining co-operation with treatment and demonstrating the effects of treatment. Continuing study of outcomes with improved methods is required, and assessment of QOL is an important part of this, part of the multi-dimensional approach to studying outcomes. This is a critical matter for future resource allocation.

\section{REFERENCES}

Abrahamson D. (1993). Institutionalisation and the long-term course of schizophrenia. British Journal of Psychiatry 162, 533-538.

Andrews G. (1999). Efficacy, effectiveness and efficiency in mental health service delivery. Australian and New Zealand Journal of Psychiaty 33, 316-322.

Atkinson M., Zibin S. \& Chuang H. (1997). Characterizing quality of life among patients with chronic mental illness: A critical examination of the self-report methodology. American Journal of Psychiatry 154, 99-105.

Awad A.G. \& Hogan T.P. (1994). Subjective response to neuroleptics and the quality of life: implications for treatment outcome. Acta Psychiatrica Scandinavica 89, Suppl. 380, 27-32.

Barry M.M. \& Crosby C. (1996). Quality of life as an evaluative measure in assessing the impact of community care on people with longterm psychiatric disorders. British Journal of Psychiatry 168, 210 216.

Barry M.M. \& Zissi A. (1997). Quality of life as an outcome measure in evaluating mental health services: a review of the empirical evidence. Social Psychiatry and Psychiatric Epidemiology 32, 3847.

Bobes J. \& Gonzalez M.P. (1997). Quality of life in schizophrenia. In Quality of Life in Mental Disorders (ed. H. Katschnig, H. Freeman and N. Sartorius), pp. 165-178. John Wiley: Chichester.

Chisholm D., Healey A. \& Knapp M. (1997). QALYs and mental health care. Social Psychiatry and Psychiatric Epidemiology 32, 68-75.

Corrigan P.W. \& Buican B. (1995). The construct validity of subjective quality of life for the severely mentally ill. Journal of Nervous and Mental Disorders 183, 281-285.

Davidson L. \& McGlashan T.H. (1997). Treated outcomes in severe and enduring mental disorders. Current Opinion in Psychiatry 10 , 183-186.

Hawthorne G., Richardson J. \& Osborne R. (1999). The Assessment of Quality of Life (AQOL) instrument: a psychometric measure of health-related quality of life. Quality of Life Research 8, 209-224.

Jenkins R. (1996). Measuring outcomes in mental health: implications for policy. In Mental Health Outcome Measures (ed. G. Thornicroft and M. Tansella). Springer-Verlag: Heidelberg.

Katschnig H. (1996). How useful is the concept of quality of life in psychiatry? Current Opinion in Psychiatry 10, 337-345.

Kavanagh S. \& Stewart A. (1995). Economic evaluations of mental health care: modes and methods. In The Economic Evaluation of Mental Health Care (ed. M.R.J. Knapp), pp. 27-60. Ashgate: Aldershot.

Leff J., Thornicroft G., Coxhead N. \& Crawford C. (1994). The TA PS Project, 22: A five-year follow-up of long-stay psychiatric patients discharged to the community. British Journal of Psychiatry $165,13-17$.

Lehman A.F. (1996). Measures of quality of life among persons with severe and persistent mental disorders. Social Psychiatry and Psychiatric Epidemiology 31, 78-88.

Mechanic D., McAlpine D., Rosenfield S. \& Davies D. (1994). Effects of illness attribution and depression on the quality of life among persons with serious mental illness. Social Science and Medicine 39,155-164.

Murray C.J.L. \& Lopez, A.D. (1996). The Global Burden of Disease: A Comprehensive Assessment of Mortality and Disability from Diseases, Injuries and Risk Factors in 1990 and Projected to 2020. Harvard University Press: Cambridge, MA.

Oliver J.P.J., Huxley P.J., Priebe S. \& Kaiser W. (1997). Measuring the quality of life of severely mentally ill people using the Lancashire Quality of Life Profile. Social Psychiatry and Psychiatric Epidemiology 32, 76-83.

Orley J., Saxena S. \& Herrman H. (1998). Quality of life and mental illness. Reflections from the perspective of the WHOQOL. British Journal of Psychiatry 172, 291-293.

Revicki, D.A. (1996). Relationship of pharmacoeconomics and healthrelated quality of life. In Quality of Life and Pharmacoeconomics in Clinical Trials, 2nd. ed. (ed. B. Spilker), pp. 1077-1083. Lippincott-Raven: Philadelphia.

Russo J., Roy-Byme P., Reeder D., Alexander M., Dwyer-O'Connor E., Dagadakis C., Ries R. \& Patrick D. (1997). Longitudinal assessment of quality of life in acute psychiatric inpatients: reliability and validity. Journal of Nervous and Mental Disorders 185 , 166-175.

Sainfort F., Becker M. \& Diamond R: (1996). Judgments of quality of life of individuals with severe mental disorders: patient self-report versus provider perspectives. American Journal of Psychiatry 153, 497-502.

Sartorius N. (1990). A WHO method for the assessment of health-related quality of life (WHOQOL). In Quality of Life Assessment: Key Issues in the 1990s (ed. S.R. Walker and R.M. Rosser), pp. 201 207. Kluwer Academic: Dordrecht.

Skantze K., Malm U., Dencker S.J., May P.R. \& Corrigan P. (1992), Comparison of quality of life with standard of living in schizophrenic out-patients. British Journal of Psychiatry 161, 797-801.

Strauss J. (1989). Subjective experiences of schizophrenia: toward a new dynamic psychiatry II. Schizophrenia Bulletin 15, 179-187.

Szmukler G.I., Burgess P., Herrman H., Benson A., Colusa S. \& Bloch S. (1996). Caring for relatives with serious mental illness: the development of the Experience of Caregiving Inventory. Social Psychiatry and Psychiatric Epidemiology 31,137-148.

Thomicroft G. \& Tansella M. (Ed.)(1996). Mental Health Outcome Measures. Springer-Verlag: Heidelberg.

Trauer T., Duckmanton R.A. \& Chiu E. (1998). A study of the quality of life of the severely mentally ill. International Journal of Social Psychiatry 44, 79-91. 
Üstün T.B. \& Chatterji S. (1998). Editorial, measuring functioning and disability a common framewark. International Journal of Methods in Psychiatric Research 7, 79-83.

Veruganti L., Heslequave R., Awad A.G. \& Seeman M.V. (1998). Quality of life mearurement in schizophrenia: reconciling the quest subjectivity with the question of reliability. Psychological Medicine 28, 165-172.

WHOQOL Group (1998a). The World Health Organization Quality of
Life Assessment (WHOQOL): Development and general psychometric properties. Social Science and Medicine 46,1569-1585. WHOQOL Group (1998b). Development of the World Health Organization WHOQOL-BREF Quality of Life Assessment. Psychological Medicine 28, 551-558.

Wilkinson G., Williams B., Krekorian H., McLees S. \& Falloon (1992), QALYs in mental health: a case study. Psychological Medicine 22 , 725-731. 